ORIGINAL

\title{
ALIMENTAÇÃO DO LACTENTE PORTADOR DE LESÃO LÁBIO-PALATAL: ALEITAMENTO E INTRODUÇÃO ALIMENTAR
}

\section{NOURISHMENT OF THE INFANT WITH CLEFT LIP AND PALATE LACTANT: NURSING AND NOURISHING INTRODUCTION}

\author{
Juliana Genovez PINI' \\ Suely Prieto de Barros Almeida PERES²
}

RESUMO

\begin{abstract}
São inquestionáveis as vantagens do leite materno para o neonato. Na sua impossibilidade, é necessária a introdução do leite artificial e de alimentação adequada como forma de reposição de nutrientes, respeitando-se a maturação neuro-fisiológica da criança. Para o estudo, foram investigadas 60 crianças de 2 a 4 anos, de ambos os sexos, portadoras de lesão lábio-palatal, pacientes do Hospital de Reabilitação de Anomalias Craniofaciais, Universidade de São Paulo, Bauru, quanto a sua história dietética pregressa (aleitamento e introdução alimentar) e peso e estatura atuais, a fim de verificar possíveis influências no estado nutricional relacionadas à sua alimentação no primeiro ano de vida. Os dados obtidos mostraram que o aleitamento materno foi significativo, e que o desmame, o aleitamento artificial e a introdução alimentar, apesar de inadequados, pouco influenciaram na antropometria atual.
\end{abstract}

Termos de indexação: lesão lábio-palatal, alimentação do lactente, aleitamento materno, introdução alimentar, lábio, lactentes.

\begin{abstract}
The advantages of the mother's milk for the newborn infants are unquestionable. In its impossibility, it is necessary the introduction of the artificial milk and adequate food as an alternative way of replacing nutrients, respecting the child's neurophysiological maturing. For this study, 60 children aged 2 to 4 years old (male and female), carriers of cleft lip and palate, who were patients of the Hospital de Reabilitação de Anomalias Craniofaciais at the Universidade de São Paulo in the city of Bauru, were investigated, considering their earlier dietary history (nursing and nourishing introduction) and present weight and height, in order to verify possible influences on nutritional status related to their feeding in the first year of life. The obtained data showed that the nursing was significant, and that the weaning, the artificial nursing and the nourishing introduction, in spite of inadequate, hardly influenced the current anthropometry.
\end{abstract}

Index terms: cleft lip-palate feeding, nutritional status, breast feeding, food introduction, lip, infant.

\footnotetext{
(1) Curso de Especialização em Nutrição Clínica e Hospitalar, Hospital de Reabilitação de Anomalias Craniofaciais, Universidade de São Paulo (HRAC-USP). Rua João Henrique Dix, 1-35, 17063-220, Bauru, SP, Brasil.

(2) Serviço de Nutrição e Dietética, Hospital de Reabilitação de Anomalias Craniofaciais, Universidade de São Paulo (HRAC-USP). Rua Silvio Marchiori, 3-20, 17043-900, Bauru, SP, Brasil. Correspondência para/Correspondence to: S.P.B.A. PERES.
} 


\section{INTRODUÇÃO}

A lesão lábio-palatal é uma malformação congênita, com incidência de 1 para 650 nascimentos (Souza-Freitas, 1974), que ocorre quando não há fusão do lábio e/ou palato durante o período intra-uterino (Kelts \& Jones, 1984). De modo geral, segundo Spina et al. (1972), as lesões estão divididas em três grupos: fissura Pré-Forame Incisivo (ou isolada de lábio), fissura Pós-Forame Incisivo (ou isolada de palato) e fissura Transforame Incisivo (ou de lábio e palato) (Figura 1).

As fissuras, principalmente as de palato, causam dificuldades alimentares levando, muitas vezes, à desnutrição. Com o processo de sucção prejudicado, a quantidade dos alimentos ingerida nem sempre é suficiente para suprir as necessidades do bebê, resultando em pouco ganho ponderal. Este quadro é agravado pelo escape de alimentos pelo nariz e ingestão excessiva de ar que provocam vômitos, engasgos e perdas de alimentos. Além disto, são comuns as infecções de ouvido e as pneumonias aspirativas (Paradise \& McWilliams, 1974).

No entanto, é importante colocar que a lesão lábio- palatal não exclui o aleitamento materno. Almeida \& Passeroti (1986), relatam que o aleitamento materno é mais satisfatório em crianças com fissura menos complexa, entretanto, Styer \& Freeh (1981), mostram que as fissuras de modo geral não o impedem de ser feito, inclusive recomendam que o bebê seja amamentado pelo próprio valor nutricional do leite, pelo desenvolvimento dos músculos da face pela sucção, além de favorecer a relação mãe-filho.

Sabe-se que crianças amamentadas ao seio adoecem com menos freqüência, pois a mãe transmite fatores de defesa ao filho através do colostro e do leite (Barros et al., 1982; Mandarino \& Oliveira, 1982; Andrade \& Torres, 1985; Goldman \& Goldblum, 1991; Euclydes, 1997). Importantes efeitos benéficos do leite sobre o desenvolvimento cognitivo e a saúde bucal da criança também são descritos (Moraes, 1998; Vieira, 1998). Na impossibilidade do aleitamento materno, deve-se orientar a introdução do leite mais adequado a cada situação (Fomon, 1993). Geralmente são fórmulas pasteurizadas (leites de vaca e cabra fluidos) ou fórmulas industrializadas (leites em pó) e a introdução de novos alimentos deve ser feita respeitando-se a maturação neuro-fisiológica da criança, bem como suas necessidades nutricionais (Ornellas \& Ornellas, 1983; Rowland, 1985; Pernetta, 1988).

O presente trabalho visa avaliar a alimentação de crianças portadoras de lesão lábio-palatal, de dois a quatro anos, ambos os sexos, quanto ao tipo de aleitamento e à introdução alimentar recebidos do nascimento ao primeiro ano de vida, verificando possíveis influências no seu estado nutricional atual.

\section{CASUÍSTICA E MÉTODOS}

Foram avaliadas 60 crianças de 2 a 4 anos de idade, ambos os sexos, portadoras de lesão de lábio e/ou palato, atendidas no ambulatório do Hospital de Reabilitação de Anomalias Craniofaciais, Universidade de São Paulo, Bauru, SP, no período de agosto de 1998 a abril de 1999.

Foi realizada uma investigação da história dietética

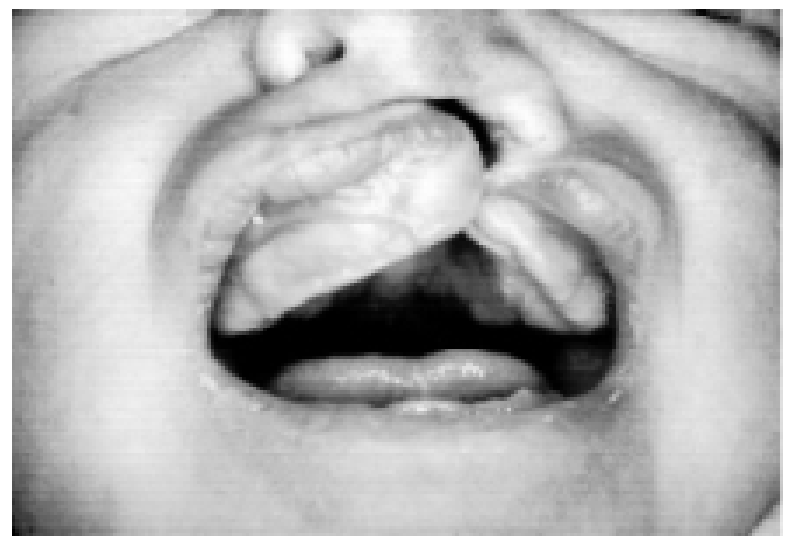

FISSURA PRÉ-FORAME INCISIVO UNILATERAL

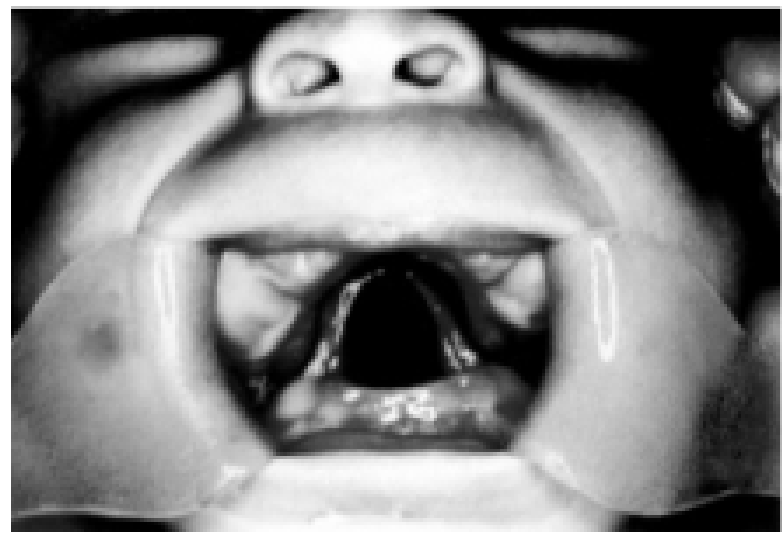

FISSURA PÓS-FORAME INCISIVO TOTAL

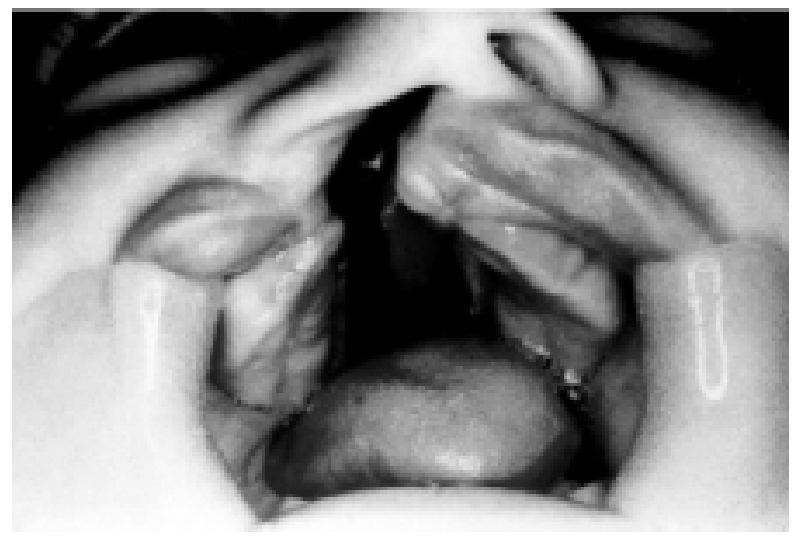

FISSURA TRANSFORAME INCISIVO UNILATERAL

Figura 1. Lactentes portadores de lesões lábio e/ou palatais. 
pregressa do paciente (do nascimento aos 12 meses de idade), aplicando-se um questionário à mãe da criança com dados sobre o aleitamento (tipo e diluição dos leites artificiais) e a introdução alimentar (tipo de alimento e época da introdução).

Como padrão de referência para aleitamento artificial utilizou-se o esquema proposto por Peres (1999) o qual recomenda o uso de leite fluido diluído ao meio ou leite em pó integral a 7,5\% para crianças até 4,5 kg de peso; leite fluido diluído a dois terços ou leite em pó integral a $10,0 \%$ até os $7 \mathrm{~kg}$ e leite fluido sem diluir ou em pó integral a $15,0 \%$ a partir dos $7 \mathrm{~kg}$. No caso das fórmulas industrializadas específicas para menores de 6 meses, a diluição será de acordo com o rótulo do produto.

Para introdução de alimentos também se utilizou o proposto por Peres (1999), de acordo com o tipo de leite recebido pela criança. Desta forma, quando em aleitamento materno exclusivo, o suco e a papa de frutas são introduzidos entre o quarto e o sexto meses de idade e a papa de legumes, carne e cereais no sétimo mês. Quando em aleitamento artificial, o suco de frutas é introduzido aos dois meses de idade, a papa de frutas aos três meses e a papa de legumes, carne e cereais aos quatro meses. As leguminosas são sempre oferecidas após o oitavo mês de vida e, em torno de um ano, a criança deve estar ingerindo a alimentação da família.

Após a investigação dietética, foi feita uma avaliação antropométrica que constou de medidas de Peso (P) e Estatura (E). O peso foi aferido em balança tipo plataforma, com o paciente descalço, apenas com roupa íntima. A estatura foi verificada em antropômetro com o paciente em pé e descalço, com as costas, nádegas e calcanhares encostados na haste rígida e com o esquadro sobre sua cabeça. Tais dados foram relacionados à idade utilizando-se a classificação de Waterlow-Batista, com a terminologia de Batista-Filho (Viacava et al., 1983).
Os pacientes foram classificados sócioeconomicamente segundo o proposto por Graciano et al. (1996) e aplicou-se o teste " $\mathrm{t}$ " de student para comparação da amostra por idade por sexo (Curi, 1998).

\section{RESULTADOS}

A amostra foi formada por $55,0 \%$ de crianças do sexo masculino e $45,0 \%$ do feminino não havendo diferenças estatísticas entre as idades $(P>0,05)$ que foi de $35,76 \pm 6,61$ meses para o sexo masculino e 34,93 $\pm 6,16$ meses para o sexo feminino, sendo que $86,7 \%$ dos pacientes pertenciam ao nível socioeconômico baixo e $13,3 \%$ ao médio.

A lesão lábio-palatal predominante foi a Transforame Incisivo seguida da Pós-forame Incisivo. Do total da amostra, 33 (55\%) receberam leite materno e 27 (45\%) leite artificial. O maior número de pacientes em aleitamento materno era portador de fissura Transforame Incisivo mas aleitaram ao seio por poucos dias. As crianças que o fizeram por maiores períodos de tempo foram as portadoras de fissura Pré-forame Incisivo, com uma média de 332,5 $\pm 45,9$ dias de aleitamento (Tabela1).

Fórmula láctea industrializada foi a mais utilizada pela maioria dos pacientes, tanto pelos que não aleitaram ao seio como pelos demais, após o desmame. Um paciente recebeu leite de cabra fluido após o desmame (Tabela 2).

Com relação à diluição da fórmula láctea utilizada, $41,7 \%$ receberam leite com diluição correta para a idade e 58,3\% com diluição incorreta.

Ao analisar-se a época de introdução dos demais alimentos, verificou-se que a maioria dos pacientes teve uma introdução tardia de suco, frutas, hortaliças e cereais e precoce das leguminosas. Entretanto, em relação à introdução da alimentação da família, a época foi adequada para a grande maioria dos pacientes (Tabela 3).

Tabela 1. Classificação dos pacientes por tipo de fissura e sua relação com o aleitamento materno.

\begin{tabular}{|c|c|c|c|c|c|c|c|c|c|c|}
\hline \multirow[t]{2}{*}{ Variáveis } & \multicolumn{2}{|c|}{$\begin{array}{l}\text { Pré-Forame } \\
\text { Incisivo }\end{array}$} & \multicolumn{2}{|c|}{$\begin{array}{l}\text { Pós-Forame } \\
\text { Incisivo }\end{array}$} & \multicolumn{2}{|c|}{$\begin{array}{c}\text { Pré + Pós-Forame } \\
\text { Incisivo }\end{array}$} & \multicolumn{2}{|c|}{$\begin{array}{c}\text { Transforame } \\
\text { Incisivo }\end{array}$} & \multicolumn{2}{|c|}{ Total } \\
\hline & $\mathrm{n}$ & $\%$ & $\mathrm{n}$ & $\%$ & $\mathrm{n}$ & $\%$ & $\mathrm{n}$ & $\%$ & $\mathrm{n}$ & $\%$ \\
\hline Total de pacientes por tipo de fissura & 3 & 5,0 & 17 & 28,3 & 6 & 10,0 & 34 & 56,7 & 60 & 100 \\
\hline Pacientes em aleitamento materno & 2 & 3,3 & 11 & 18,3 & 3 & 5,0 & 17 & 28,3 & 33 & 55 \\
\hline Duração do aleitamento materno em dias & \multicolumn{2}{|c|}{$332,5 \pm 45,9^{*}$} & \multicolumn{2}{|c|}{$82,8 \pm 53,7^{*}$} & \multicolumn{2}{|c|}{$50,0 \pm 17,3^{*}$} & \multicolumn{2}{|c|}{$42,9 \pm 30,0^{*}$} & \multicolumn{2}{|c|}{ - } \\
\hline
\end{tabular}

$\left({ }^{*}\right)$ Média \pm Desvio-Padrão.

Tabela 2. Tipos de leite ingeridos pelos pacientes.

\begin{tabular}{|c|c|c|c|c|}
\hline & \multicolumn{2}{|c|}{ Pacientes em desmame do leite materno $(n=33)$} & \multicolumn{2}{|c|}{ Pacientes em aleitamento artificial $(n=27)$} \\
\hline & $\mathrm{n}$ & $\%$ & $\mathrm{n}$ & $\%$ \\
\hline Fórmulas industrializadas & 21 & 63,6 & 20 & 74,1 \\
\hline Leite fluido de vaca & 11 & 33,3 & 7 & 25,9 \\
\hline Leite fluido de cabra & 1 & 3,1 & - & - \\
\hline
\end{tabular}


Tabela 3. Adequação da época de intodução dos alimentos, segundo PERES (1999).

\begin{tabular}{|c|c|c|c|c|c|c|}
\hline \multirow{3}{*}{ Variáveis } & \multicolumn{6}{|c|}{ Época de Introdução } \\
\hline & \multicolumn{2}{|c|}{ Recomendada } & \multicolumn{2}{|c|}{ Após recomendado } & \multicolumn{2}{|c|}{ Antes Recomendado } \\
\hline & $\mathrm{n}$ & $\%$ & $\mathrm{n}$ & $\%$ & $\mathrm{n}$ & $\%$ \\
\hline Fruta & 12 & 20,00 & 43 & 71,67 & 5 & 8,33 \\
\hline Verdura + Legume + Carne & 17 & 28,33 & 33 & 55,00 & 10 & 16,67 \\
\hline Cereal & 10 & 16,67 & 27 & 45,00 & 23 & 38,33 \\
\hline Leguminosa & 9 & 15,00 & 22 & 36,67 & 29 & 48,33 \\
\hline Alimentação família & 43 & 71,67 & 17 & 28,33 & - & \\
\hline
\end{tabular}

Em relação à avaliação antropométrica atual, 70\% dos pacientes foram classificados como eutróficos, $20 \%$ como desnutridos atuais e 10\% como desnutridos crônicos.

\section{DISCUSSÃO}

A amostra mostrou-se homogênea quanto ao sexo e à idade por sexo. Predominou-se o nível socioeconômico baixo e a fissura Transforame Incisivo.

Observou-se que a maioria dos pacientes estudados foram aleitados ao seio, o que está de acordo com Styer \& Freeh (1981), que colocaram que as fissuras, de modo geral, não impedem o aleitamento materno.

Quando separados por tipo de lesão, os resultados mostraram que os portadores de fissura Pré-Forame Incisivo conseguiram ser amamentados por um maior período de tempo, o que também foi observado nos estudos de Kelts \& Jones (1984) e de Almeida \& Passerotti (1986), mostrando que o aleitamento materno é mais efetivo nas fissuras mais simples. No entanto, foi importante o número de crianças com fissura Transforame Incisivo aleitadas ao seio, uma vez que a gravidade da própria lesão dificulta o processo.

Com relação ao aleitamento artificial, a maioria fez uso de fórmulas lácteas industrializadas. Kelts \& Jones (1984), preconizam fórmula láctea industrializada específica para menores de seis meses, como o melhor substituto do leite materno e, Euclydes (1997), reforça em seus estudos a improbidade do leite de vaca no primeiro ano de vida por estar relacionado a problemas como deficiência de ferro e perda de sangue pelas fezes, aumentar o risco de alergia, conter carga elevada de solutos e ser deficiente em ácido linoléico. Apesar disto o leite de vaca fluido foi bastante utilizado no presente trabalho, provavelmente devido ao baixo nível socioeconômico predominante na amostra.

Apesar de Woiski (1988) e Peres (1999) terem demonstrado a importância da diluição correta do leite artificial na prevenção de estados de desnutrição, grande parte dos pacientes estudados receberam leite com diluição incorreta em algum momento do estudo, o que pode ser justificado pelo nível socioeconômico baixo a que pertenciam a maioria destas crianças. Segundo Euclydes (1997), o nível socioeconômico geralmente impede que um leite com composição mais adequada seja oferecido à criança ou, mesmo quando comprado, muitas vezes é preparado inadequadamente.

De forma geral, a introdução de alimentos como sucos, frutas, verduras, legumes, cereais e leguminosas foi feita em épocas inadequadas (precoce ou tardiamente) e, somente a passagem para alimentação da família foi adequada para a maioria dos pacientes. Observou-se, portanto que, durante o primeiro ano de vida dos pacientes estudados, predominaram erros na época de introdução de alimentos e na diluição correta dos leites artificiais utilizados e, ao procurar relacionar-se tais inadequações com o estado nutricional atual dos pacientes, verificou-se pouca influência da mesma, uma vez que a maior parte dos pacientes da amostra encontrava-se eutrófica. O reflexo talvez seja sentido nos $10 \%$ de desnutridos crônicos.

\section{CONCLUSÃO}

Conclui-se, portanto, que apesar das crianças serem portadoras de malformações que possam comprometer sua sucção, conseguiram aleitar ao seio nos primeiros meses de vida, o que reforçou a importância do incentivo ao aleitamento materno, mesmo nas fissuras de maior complexidade. E, mesmo detectando-se erros na introdução alimentar e na diluição dos leites artificiais durante o primeiro ano de vida, não houve repercussão significativa na avaliação antropométrica atual.

\section{REFERÊNCIAS BIBLIOGRÁFICAS}

ALMEIDA, M.L.G., PASSEROTTI, A.L.A.C. Amamentação materna em fissurados: estudo retrospectivo. Pediatria Moderna, São Paulo, v.21, n.2, p.85-90, 1986.

ANDRADE, M.G.N., TORRES, I.A. Algumas considerações sobre o aleitamento materno no contexto social. Ars Curandi em Odontologia, São Paulo, v.11, n.1, p.38-41, 1985. 
BARROS, M.D., KULESZA, T.M., RANNA, W., CARNEIRO-SAMPAIO, M. Papel do leite materno na defesa do lactente contra infecções. Pediatria, São Paulo, v.4, n.1, p.88-102, 1982.

CURI, P.R. Metodologia e análise da pesquisa em Ciências Biológicas. 2.ed. Botucatu : Tipomic, 1998. 363p.

EUCLYDES, M.P. Nutrição do lactente: base científica para uma alimentação adequada. Viçosa : Jard, 1997. 461p.

FOMON, S.J. Nutrition of normal infants. Saint Louis : Mosby, 1993. $475 \mathrm{p}$.

GOLDMAN, A.S., GOLDBLUM, R.M. Human milk: immunologicnutritional relationships. Annals of the New York Academy of Sciences, New York, p.236-245, 1991.

GRACIANO, M.I.G., LEHFELD, N.A.S., NEVES FILHO, A. Critérios da avaliação para classificação sócio-econômica: elementos de atualização - Parte II. Serv Soc Real, v.5, p.171-201, 1996.

KELTS, D.G., JONES, E.G. (ed.). Manual de nutrição infantil. Tradução de Hildegard Thiemann Buckup. Rio de Janeiro: Guanabara, 1984. 312p. Título original: Manual of pediatric nutrition.

MANDARINO, V., OLIVEIRA, S.M.U. Alimentação do recém-nascido a termo. Pediatria Moderna, São Paulo, v.17, n.3, p.136-138, 1982.

MORAES, E.M.F. O aleitamento materno e seus benefícios sob o ponto de vista de saúde bucal. Baby site, 1996-2000. Disponível em: <www: http://ipanema.com/babysite/artig2.htm>. Acesso em: 2 jul. 1998.

ORNELLAS, A., ORNELLAS, L.H. Alimentação da criança: nutrição aplicada. 2.ed. rev. atual. São Paulo : Atheneu, 1983. 455p.

PARADISE, J.L., McWILLIAMS, B.J. Simplified feeder for infants with cleft palate. Pediatrics, Springfield, v.53, n.4, p.566-568, 1974.
PERES, S.P.B.A. Alimentando uma criança portadora de lesão lábiopalatal: primeiro ano de vida. Nutri Vitae, São Paulo, v.2, n.2, p.45-54, 1999.

PERNETTA, C. Alimentação da criança. 8.ed. Rio de Janeiro : Guanabara, 1988. 226p.

ROWLAND, M.G. The "why" and "when" of introducing food to infants: growth in young breast-fed infants and some nutritional implications. American Journal of Clinical Nutrition, Bethesda, v.41, n.2, p.459-463, 1985. Supplement.

SOUZA-FREITAS, J.A. Centro de Pesquisa e Reabilitação de Lesões Lábio-Palatais. Bauru : Faculdade de Odontologia de Bauru, Universidade de São Paulo, 1974. 55p.

SPINA, V., PSILLAKIS, J.M., LAPA, F.S., FERREIRA, M.C. Classificação das fissuras lábio-palatais: sugestão de modificação. Revista do Hospital das Clínicas de Faculdade de Medicina da Universidade de São Paulo, São Paulo, v.27, n.1, p.5-6, 1972.

STYER, G.W., FREEH, K. Feeding infants with cleft lip and/or palate. Jogn Nursing, Philadelphia, v.10, n.5, p.329-332, 1981.

VIACAVA, F., FIGUEIREDO, C.M.P., OLIVEIRA, W.A. A desnutrição no Brasil: uma análise do estudo nacional de despesa familiar (IBGE 74/75) para o Nordeste, Estado de São Paulo e Estado do Rio de Janeiro. Petrópolis : Vozes, 1983. 199p.

VIEIRA, G.O. Indicadores do aleitamento materno na cidade de Feira de Santana, Bahia. Jornal de Pediatria, Rio de Janeiro, v.74, n.1, p.11-16, 1998.

WOISKI, J.R. Nutrição e dietética em pediatria. 3.ed. Rio de Janeiro : Atheneu, 1988. 274p.

Recebido para publicação em 11 de julho de 2000 e aceito em 10 de janeiro de 2001. 